\title{
Null Propagation of Partially Massless Higher Spins in (A)dS and Cosmological Constant Speculations
}

\author{
S. Deser and A. Waldron \\ Physics Department, Brandeis University, Waltham, MA 02454, USA \\ deser, wally@brandeis.edu
}

\begin{abstract}
We show explicitly that all partially and strictly massless fields with spins $s \leq 3$ in (A)dS have null propagation. Assuming that this property holds also for $s>3$, we derive the mass-cosmological constant tunings required to yield all higher spin partially massless theories. As $s$ increases, the unitarily allowed region for massive spins is squeezed around $\Lambda=0$, so that an infinite tower of massive particles forces vanishing $\Lambda$. We also speculate on the relevance of this result to string theory and supergravity in (A)dS backgrounds.
\end{abstract}




\section{Introduction}

The cosmological constant "problem" has seen many incarnations. Current observational evidence points to a small but positive, de Sitter (dS), value for $\Lambda$ [1], while recent theoretical advances relating gauge and gravitational theories are based on strings in $\Lambda<0$, Anti de Sitter (AdS), backgrounds [2], the sign also required by conventional cosmological supergravity [3]. In the context of $\Lambda \neq 0$ spaces, a recent analysis of free, higher $(s>1)$ spin fields has led to dramatic results [4]: In addition to the usual Minkowski possibilities of 2 strictly massless or $2 s+1$ massive propagating degrees of freedom $(\mathrm{DoF})$, there are intermediate partially massless theories corresponding to new gauge invariances for particular tunings of the mass $m^{2}$ to $\Lambda$ that remove lower helicity states from the spectrum. These novel theories divide the $\left(m^{2}, \Lambda\right)$ plane into unitarily allowed and forbidden regions. While explicit constructions were provided in [4] for all $s \leq 3$, two important questions were left open:

1. Do these new partially massless theories propagate on the null cone?

2. What $m^{2}: \Lambda$ tunings are necessary to achieve partial masslessness for arbitrary $s>3$ ?

In this Letter, we answer both questions and draw some possible consequences: Null propagation is verified for all the $s \leq 3$ gauge theories and assuming that this result persists for $s>3$ provides a simple derivation of the higher spin $m^{2}: \Lambda$ tunings. Physically, this assumption just extends the usual flat space wisdom that gauge invariance implies lightlike propagation, but there is a second compelling reason to assert its validity: The existence of partially massless theories is related directly to representations of the (A)dS spacetime algebra. A study of the $s=2$ partially massless theory [5, 6] prompted a verification that (bosonic) representations with missing lower helicities were indeed possible [7]. Our (local) models realize precisely these representations and construct their fermionic counterparts.

A potentially wide-ranging consequence of our results is that as $s$ increases, unitary massive theories can only occupy a region in the $\left(m^{2}, \Lambda\right)$ plane that is squeezed onto the line $\Lambda=0$. [Individual partially massless theories are unitary whenever their gauge invariances remove all negative norm states.] Some ramifications of this result for the cosmological constant problem and string theory in constant curvature backgrounds are also discussed. 


\section{Null Propagation of Partially Massless Bosons}

Our method is direct: We solve the helicity $\pm s$ field equations for all higher spins in a dS background

$$
\begin{gathered}
d s^{2}=-d t^{2}+f^{2}(t) d \vec{x}^{2}, \\
f(t) \equiv e^{M t}, \quad M^{2} \equiv \Lambda / 3 .
\end{gathered}
$$

When they are present in the spectrum, all lower helicities propagate in exactly the same manner (this has been worked out in great detail [4] for $s=2$ ), so we need not treat them separately. In the frame (1), spatial slices are flat, which allows the usual definition of helicity. Solutions to the field equations are of Bessel type, and take the form

$$
\text { (slowly varying }) \times \exp (i \omega u+i \vec{k} \cdot \vec{x}), \quad \omega^{2}=\vec{k}^{2},
$$

whenever the index $\nu$ of the Bessel function is half integer. Here

$$
u \equiv-\frac{f^{-1}(t)}{M}
$$

is the conformal time coordinate in terms of which

$$
d s^{2}=\frac{1}{M^{2} u^{2}}\left(-d u^{2}+d \vec{x}^{2}\right) .
$$

Therefore we can read off the theories propagating on the null cone directly from the index $\nu$. We will find null propagation for all the $s \leq 3$ partially massless theories presented in [4].

The onshell conditions for a massive spin $s$ field in (A)dS are

$$
\left(D^{2}-m^{2}-\left(2+2 s-s^{2}\right) M^{2}\right) \phi_{\mu_{1} \ldots \mu_{s}}=0, \quad D . \phi_{\mu_{2} \ldots \mu_{s}}=0=\phi_{\rho \mu_{3} \ldots \mu_{s}}^{\rho} .
$$

The parameter $m^{2}$ has been chosen so that $m^{2}=0$ corresponds to the strictly massless (i.e., helicity $\pm s$ only) theory. [For $s=0$, equation (5) describes a conformally improved scalar.]

\footnotetext{
${ }^{1}$ The Riemann tensor in constant curvature spaces is $R_{\mu \nu \rho \sigma}=-\frac{2 \Lambda}{3} g_{\mu[\rho} g_{\sigma] \nu}$. The actions of commutators of covariant derivatives are summarized by the vector-spinor example $\left[D_{\mu}, D_{\nu}\right] \psi_{\rho}=\frac{2 \Lambda}{3} g_{\rho[\mu} \psi_{\nu]}+\frac{\Lambda}{6} \gamma_{\mu \nu} \psi_{\rho}$. Our metric is "mostly plus" and Dirac matrices are "mostly hermitean".
} 
The traceless-transverse part of a spatial tensor is denoted by the superscript $t$. We project out helicity $\pm s$ by computing only the tt part of (5), which in the frame (四) reads,

$$
\left(-\frac{d^{2}}{d t^{2}}+(2 s-3) M \frac{d}{d t}+f^{-2} \vec{\partial}^{2}-m^{2}+2(s-1) M^{2}\right) \phi_{i_{1} \ldots i_{s}}^{t t}=0 .
$$

Fourier transforming $\vec{\partial} \rightarrow i \vec{k}$, changing coordinates

$$
z \equiv-\frac{k f^{-1}(t)}{M}=k u, \quad(k \equiv|\vec{k}|)
$$

and the field redefinition (suppressing indices)

$$
\phi^{t t} \equiv z^{3 / 2-s} q
$$

yield Bessel's equation

$$
\frac{d^{2} q}{d z^{2}}+\frac{1}{z} \frac{d q}{d z}+\left(1-\frac{\nu^{2}}{z^{2}}\right) q=0
$$

with index

$$
\nu^{2}=\frac{1}{4}+s(s-1)-\frac{m^{2}}{M^{2}} .
$$

We may now read off the null propagating theories.

\section{Examples}

1. Conformal Scalar: At $s=0=m$ we obtain $\nu=1 / 2$ and $q(z)=$ $z^{-1 / 2} \exp (i z)$ which implies a solution of the form (2). The value $\nu=$ $1 / 2$ also characterizes all the higher spin "conformal" theories.

2. Maxwell: In $d=4$ the $s=1, m=0$ vector theory is conformal and here $\nu=1 / 2$.

3. Spin 2: Spin 2 can be either strictly massless at $m=0$ or partially massless when $m^{2}=2 M^{2}$. The latter model, with its accompanying scalar gauge invariance, takes the conformal value $\nu=1 / 2$. Of course, the $m^{2}=0$ linearized cosmological Einstein theory also propagates on the cone, but this is achieved by the solution $\nu=3 / 2$ for which $q(z)=(z+i) z^{-3 / 2} \exp (i z)$. 
4. Spin 3: Here there are 3 possibilities; the strictly massless theory at $m=0$ for which $\nu=5 / 2\left(\Rightarrow q(z)=\left(z^{2}+3 i z-3\right) z^{-5 / 2} \exp (i z)\right)$, a partially massless one with helicities $( \pm 3, \pm 2)$ at $m^{2}=4 M^{2}$ with $\nu=$ $3 / 2$ and finally, a theory with a scalar gauge invariance and helicities $( \pm 3, \pm 2, \pm 1)$ with $m^{2}=6 M^{2}$ and the conformal value $\nu=1 / 2$. Clearly all these theories have null propagation.

Noting that the value of $\nu$ can be associated with the type of gauge invariance (for example, the conformal value $\nu=1 / 2$ always belongs to the scalar invariance), we are led to the following conjecture:

Conjecture: All partially massless higher spin bosons propagate on the null cone. The spin $s$ theory with helicities $( \pm n, \ldots, 0)$ removed appears when

$$
m^{2}=M^{2}(s(s-1)-n(n+1)),
$$

and has Bessel index $\nu=n+1 / 2$. All these gauge theories are unitary.

Note that the strictly massless theory has $n=s-1, m^{2}=0$ and $\nu=s-1 / 2$ whereas the conformal value $\nu=1 / 2$ appears for the scalar gauge invariant theory with $n=0$ and $m^{2}=M^{2} s(s-1)$. An additional strong justification for this conjecture is that these representations have been seen before in a study [7] of bosonic Laplace-Beltrami operators on dS backgrounds'.

\section{$3 \quad$ Null Propagation of Partially Massless Fermions}

Partially massless fermionic theories are found in AdS. However, we continue to work in dS because of the simplicity of the metric (11). The results for the partially massless lines depend on $\mathrm{m}^{2}$ and $\Lambda \equiv 3 M^{2}$ only and continue to AdS 3. Just as for the lowest "multiline" $s=5 / 2$ case, only the strictly

\footnotetext{
${ }^{2}$ We note that for $s \geq 5 / 2$, massive theories require auxiliary fields; these were explicitly seen to decouple in the $s=5 / 2,3$ strictly massless limits [4]. While we have no proof that this decoupling persists for higher $s$, the representations of [7] suggest this is the case.

${ }^{3}$ The cost is that, in dS, the mass parameter $m$ is pure imaginary. The action is therefore no longer hermitean, although these theories actually possess a positive norm Hilbert space. This is the higher spin generalization of the old dS supergravity conundrum discussed in [3].
} 
massless helicity $\pm s$ gauge theory is unitary in $\mathrm{AdS}$, owing to the line ordering problem discussed in $[4$.

A massive spin $s \equiv \sigma+1 / 2$ fermionic field satisfies the onshell conditions

$$
(\not D+m) \psi_{\mu_{1} \ldots \mu_{\sigma}}=0 \quad \text { D. } \psi_{\mu_{2} \ldots \mu_{\sigma}}=0=\gamma \cdot \psi_{\mu_{2} \ldots \mu_{\sigma}} .
$$

We choose the local Lorentz gauge

$$
e_{0} \underline{0}=1, \quad e_{i} \underline{j}=f(t) \delta_{i} \underline{j},
$$

where underlined indices are flattened. The Dirac equation for the (spatially transverse, gamma-traceless " $t t$ ") helicities $\pm s$ reads

$$
\left(\frac{d}{d t}+(2-s) M-f^{-1} \gamma^{0 \underline{j}} \partial_{j}-\gamma^{\underline{0}} m\right) \psi_{i_{1} \ldots i_{\sigma}}^{t t}=0 .
$$

In the usual large/small component basis (suppressing indices again)

$$
\gamma^{\underline{0}}=\left(\begin{array}{rr}
-i & 0 \\
0 & i
\end{array}\right), \quad \gamma^{\underline{j}}=\left(\begin{array}{cc}
0 & i \sigma^{j} \\
-i \sigma^{j} & 0
\end{array}\right), \quad \psi^{t t}=\left(\begin{array}{c}
\chi \\
\phi
\end{array}\right)
$$

we can eliminate the small component $\phi$ and obtain the second order equation for $\chi$

$$
\left(-\frac{d^{2}}{d t^{2}}+(2 s-5) M \frac{d}{d t}+f^{-2} \vec{\partial}^{2}-(s-2)(s-3) M^{2}-i m M-m^{2}\right) \chi=0 .
$$

The coordinate transformation (7) and field redefinition

$$
\chi \equiv z^{5 / 2-s} q,
$$

yield Bessel's equation (9) with index

$$
\nu^{2}=\frac{1}{4}-\frac{i m}{M}-\frac{m^{2}}{M^{2}}=\left(\frac{1}{2}-\frac{i m}{M}\right)^{2} .
$$

Note that $m$ itself will be imaginary for the partially massless lines when they are in $\mathrm{dS}$, so the appearance of an explicit $i m$ here is appropriate. 


\section{Examples}

1. Spin 1/2: The $m=0$ spin $1 / 2$ theory is well known to be Weyl invariant, and indeed we find $\nu=1 / 2$, the conformal value.

2. Spin 3/2: As follows from linearizing cosmological supergravity, spin 3/2 is strictly massless at $m^{2}=-M^{2}$. The choice of branch $m=i M$, justified by the results, yields $\nu=3 / 2$. This is the same value we found above for its strictly massless spin 2 superpartnerst.

3. Spin 5/2: The strictly massless theory is at $m^{2}=-4 M^{2}$; the choice $m=2 i M$ yields $\nu=5 / 2$ and null propagation. The model with a spinor gauge invariance at $m^{2}=-M^{2}$ has $\nu=3 / 2$, just as for linearized cosmological supergravity.

All the above fermionic partially massless theories propagate on the null cone. Even though the fermionic analogs of the bosonic representations [7] have yet to be completed and the above results also depended on a judicious choice for the branch of the square root $\sqrt{-M^{2}}$, we make the following conjecture:

Conjecture: All partially massless fermions propagate on the null cone. The spin $s$ theory with helicities $( \pm(n+1 / 2), \ldots, \pm 1 / 2)$ removed appears at

$$
m^{2}=-M^{2}(n+1)^{2}
$$

with Bessel index $\nu=3 / 2+n$. The Hilbert space of these theories is unitary in $\mathrm{dS}$, where their actions are not hermitean.

In particular, the strictly massless theory with $n=s-3 / 2$ occurs at $m^{2}=-M^{2}(s-1 / 2)^{2}$ for $s>1 / 2$ : Its Hilbert space is unitary for any $\Lambda$, but hermiticity of its action is lost in $\mathrm{dS}$. The conformal value $\nu=1 / 2$ is only attained by the $m=0$ spin $1 / 2$ theory.

\footnotetext{
${ }^{4}$ We thank K. Peeters for a clarifying discussion on this issue.

${ }^{5}$ The argument of [5] shows that spin $3 / 2$ cannot take the conformal value $\nu=1 / 2$. This does not imply that strictly massless spin $3 / 2$ propagates off-cone, since null propagation is achieved there by $\nu=3 / 2$. This null propagation was already proven in $[8]$.
} 


\section{Cosmological Speculations}

Having presented an expeditious determination of the slopes of all partially massless lines, we now present some speculations based on the results:

\section{The Cosmological Constant Problem}

Previous attempts to render $\Lambda$ small or vanishing have been of two types: (i) Those based on (unbroken) symmetries, such as the cancellation of supersymmetric zero point energies [9] or the necessary absence of a cosmological terms in $d=11$ supergravity [10]. (ii) A dynamical solution based on quantum gravity loop corrections driving $\Lambda$ to zero [11]. Our idea is rather different: it depends only on the kinematics of a tower of free fields. Such a leap of faith should not be foreign to string theorists, since massive string states couple an infinite tower of higher spins.

The argument is that the region of the $\left(m^{2}, \Lambda\right)$ plane where the entire tower has unitary content only is squeezed onto the $\Lambda=0$ axis: The unitary region for massive higher spins in the $\left(\mathrm{m}^{2}, \Lambda\right)$ plane is bounded below in AdS by the strictly massless fermionic line

$$
m^{2}=-\frac{\Lambda(s-1 / 2)^{2}}{3}
$$

and above by the lowest partially massless bosonic gauge line (the one excluding helicity 0 ),

$$
m^{2}=\frac{\Lambda s(s-1)}{3} .
$$

Therefore the unitarily allowed region is pinched around $\Lambda=0$ for large values of $s$. Of course the robustness of this mechanism will be challenged by the usual array of difficulties introduced by interactions.

\section{String Theory}

Low energy effective field theories are obtained from strings by integrating out massive string states. Is the existence of new partially massless theories in cosmological backgrounds relevant to the quantization of strings in these spaces? 


\section{Supergravities}

A useful confirmation of our calculation was that both strictly massless spin $3 / 2$ and spin 2 had the same Bessel index, $\nu=3 / 2$, a result that ought be guaranteed by supersymmetry. Can one find other higher spin supermultiplets with equal values of $\nu$ as the basis of new locally supersymmetric theories in cosmological backgrounds? In the same highly speculative vein, we note that the purely imaginary value of $m$ for the dS partially massless fermionic theories turned out to ensure real values of the index $\nu$. Could this remark be relevant to defining a consistent cosmological supergravity theory within the dS horizon?

\section{Acknowledgments}

We thank A. Higuchi, K. Peeters, R. Woodard and M. Zamaklar for enlightening discussions. This work was supported by the National Science Foundation under grant PHY99-73935.

\section{References}

[1] See for example S. M. Carroll, "The cosmological constant," astroph/0004075.

[2] J. Maldacena, Adv. Theor. Math. Phys. 2, 231 (1998) [Int. J. Theor. Phys. 38, 1113 (1998)] [hep-th/9711200]. For a review, see O. Aharony, S. S. Gubser, J. Maldacena, H. Ooguri and Y. Oz, Phys. Rept. 323, 183 (2000) hep-th/9905111.

[3] K. Pilch, P. van Nieuwenhuizen and M. F. Sohnius, Commun. Math. Phys. 98, 105 (1985).

[4] S. Deser and A. Waldron, Phys. Rev. Lett., to appear, hep-th/0102166; Nucl. Phys. B, to appear, hep-th/0103198; Phys. Lett. B, to appear, hep-th/0103255.

[5] S. Deser and R. I. Nepomechie, Phys. Lett. B 132, 321 (1983); Annals Phys. 154, 396 (1984).

[6] A. Higuchi, Nucl. Phys. B 282, 397 (1987); ibid 325, 745 (1989); 
[7] A. Higuchi, J. Math. Phys. 28, 1553 (1987). See also U. Ottoson, Commun. Math. Phys. 8, 228 (1968); M. A. Neumark, Am. Math. Soc. Transl. (2) 6, 337 (1957); H. Thomas, Am. Math. 42, 113 (1941); J. Dixmier, Bull. Soc. Math. France 89, 9 (1961); J.G. Kuriyan, N. Mukunda and E.C.G. Sudarshan, Commun. Math. Phys. 8, 204 (1968).

[8] S. Deser and B. Zumino, Phys. Rev. Lett. 38, 1433 (1977).

[9] B. Zumino, Nucl. Phys. B 89, 535 (1975).

[10] K. Bautier, S. Deser, M. Henneaux and D. Seminara, Phys. Lett. B 406, 49 (1997) hep-th/9704131.

[11] N. C. Tsamis and R. P. Woodard, Phys. Lett. B 301, 351 (1993); Annals Phys. 238, 1 (1995). 